\title{
A Self-Tuning Receiver-Initiated MAC Protocol for Wireless Sensor Networks
}

\author{
Miguel Rodríguez-Pérez, Member, IEEE, Sergio Herrería-Alonso, \\ Manuel Fernández-Veiga, Senior Member, IEEE, and Cándido López-García
}

\begin{abstract}
Receiver-initiated medium access control protocols for wireless sensor networks are theoretically able to adapt to changing network conditions in a distributed manner. However, existing algorithms rely on fixed beacon rates at each receiver. We present a new received initiated MAC protocol that adapts the beacon rate at each receiver to its actual traffic load. Our proposal uses a computationally inexpensive formula for calculating the optimum beacon rate that minimizes network energy consumption and, so, it can be easily adopted by receivers. Simulation results show that our proposal reduces collisions and diminishes delivery time maintaining a low duty cycle.
\end{abstract}

Index Terms-Sensor Networks, Received Initiated MAC, Rate adaptation

\section{INTRODUCTION}

Medium access control (MAC) protocols for wireless sensor networks (WSN) aim to optimize power consumption in addition to other traditional design concerns of MAC schemes, as throughput or delay. Actually, the objective is that transmitters and receivers (listeners) agree on a rendezvous point that minimizes the time during which their radios are active. MAC protocols for WSNs can be broadly divided in two groups: globally synchronized, where a centralized entity orchestrates the transmission opportunities for each device, and asynchronous protocols where nodes act independently of any central entity. Asynchronous schemes can support local adaptation to varying traffic load in different parts of the network without relying on a global transmission schedule. Within this class, there exist both sender- and receiver-initiated MAC protocols. The latter family, known to be generally more network efficient [1], [2], [3], is the focus of this work.

In receiver-initiated MAC protocols, transmissions are regulated by receiving nodes. Whenever a sender has data for a neighbor node, it monitors the channel waiting for the reception of a short beacon broadcasted by the desired receiver. The beacon signs that the receiver is currently active and listening the channel, so the transmission can proceed. During this waiting time, sending nodes have to keep their radios on to detect the beacon transmission, wasting energy. Another waste of energy happens if two close senders receive a beacon in the same time interval, as their transmission will collide, even if the beacons came from different receivers. PW-MAC [4] deals with both problems by transmitting the beacons at times predictable by the senders. The receivers randomize their interbeacon period with a pseudo-random number generator, whose

The authors are with the Telematics Engineering Dept., Univ. of Vigo, 36310 Vigo, Spain. email: miguel@ det.uvigo.es (M. Rodríguez-Pérez). (C) 2015 IEEE. Personal use of this material is permitted. Permission from IEEE must be obtained for all other uses, in any current or future media, including reprinting/republishing this material for advertising or promotional purposes, creating new collective works, for resale or redistribution to servers or lists, or reuse of any copyrighted component of this work in other works. DOI: 10.1109/LWC.2015.2472398 parameters are sent in every beacon. Thus, after hearing a first beacon, a sender can find out all the future beacon transmission times and avoid to monitor the channel continuously. Thus, the radio of the sender can be turned on just a short time before the next expected beacon transmission.

Clearly, PW-MAC cannot adapt to a variable traffic load unless its randomized inter-beacon times respond to changes in time and space. In multi-hop sensor networks the load varies with time and also with location, since nodes closer to the sinks aggregate traffic from farther senders. An energyefficient MAC protocol should modulate the beacon interval and preserve a duty cycle as low as that in PW-MAC.

In this paper, we build on PW-MAC and present a receiverinitiated MAC protocol for WSNs able to generate the beacons in a predictable yet adaptive, load-dependent way. We also derive the optimum beacon rate that minimizes the energy consumption in the network, given a traffic load. The optimum rate follows a simple formula which can be easily implemented in computationally constrained sensors. The rest of the paper is organized as follows. Section II presents a complete description of our enhancements to existing receiver-initiated MAC algorithms. We present experimental results in Section III. Finally, the conclusions are summarized in Section IV.

\section{PROTOCOL DESCRIPTION}

As stated in the Introduction, our work builds on the PW-MAC [4] protocol for its basic operation. Like in other receiver-initiated MAC protocols, the receivers transmit beacon frames so that neighbor nodes with pending traffic can send their data. PW-MAC uses a pseudo-random sequence known to every node in the network for scheduling its beacon transmission times. De-synchronizing the beacons reduces collisions, while making their actual transmission time predictable avoids increasing the duty cycle. Notice that, for randomizing the beacons, a pseudo-random generator of high statistical quality is not necessary, and a description of its seed can occupy very few bytes in the frame header. For instance, PW-MAC uses just six bytes in the beacon to transmit the parameters of the linear congruential generator (LCG) used to generate the inter-beacon times.

If adaptation to different traffic incoming rates is desired, the beacon rate cannot remain static. In particular, when the traffic load increases, the number of beacons generated in a given time interval must be increased adding new extra beacons. However, changing the beacon frequency proportionally to the traffic load is naive and fails to work properly, because all the 


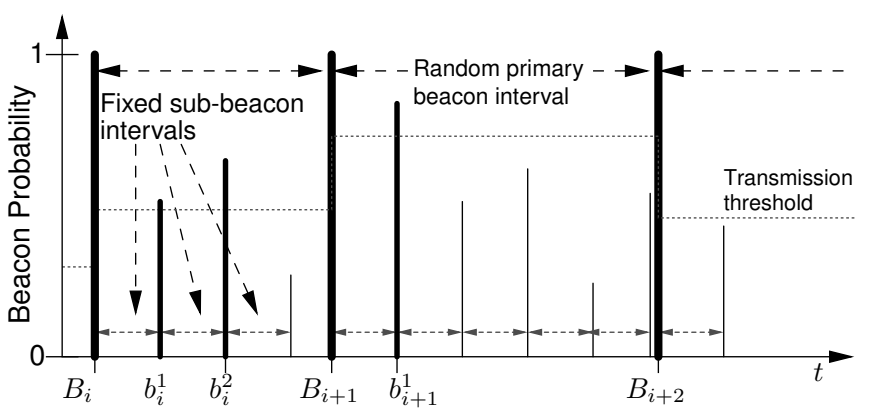

Figure 1. Predictable sub-beacon generation procedure. Actually transmitted sub-beacons appear in bold lines. In the example $n_{\mathrm{b}}=4$. For $t \in\left[B_{i}, B_{i+1}\right)$, $f=3$ so $t h_{\mathrm{b}}=0.5$. For $t \in\left[B_{i+1}, B_{i+2}\right), f=2$ so $t h_{\mathrm{b}}=0.75$.

nodes in the network - active or currently inactive- should be notified synchronously. Otherwise, non-listening neighbors (neighbors with no data for the receiver when the beacon is transmitted) would be unaware of the changes and would fail to meet at future rendezvous points. Consequently, the extra beacons (in the following, sub-beacons) must be independent of the regular or primary beacons.

One way to schedule the extra beacons is to divide the interval between two primary beacons in $f$ subintervals, where $f$ is a speeding factor, and transmit extra beacons between these subintervals. The speeding factor can be announced in the regular beacons without disturbing the operations of nonlistening nodes. Since changes to $f$ are likely to be sporadic, the nodes could assume that the speeding factor stays in effect indefinitely. However, this assumption poses a problem when the speeding factor changes (even if it is incremented): the boundaries between the subintervals change wildly, and the nodes that lack updated information cannot thereafter predict correctly the transmission times of the sub-beacons.

In the rest of this Section, we describe how to modify PWMAC to accomplish rate-adaptation. We will however ignore the details concerning the clock adjustments among nodes and the collision resolution protocol, for their behavior in $\mathrm{PW}$ MAC applies unmodified to our proposal.

\section{A. Generating Predictable Sub-beacons}

We have resorted to fix the transmission times of the subbeacons, but make their actual transmissions dependent on the speeding factor. To this end, let us divide the inter-beacon interval into fixed intervals in which a sub-beacon could potentially be transmitted. The actual transmission of each individual sub-beacon is going to depend on the speeding factor $f$ and it will be monotone, i.e., if a sub-beacon is transmitted for a given factor $f$, then it will be also transmitted when the speeding factor increases. This assumption allows uninformed senders to predict the future sub-beacons even in case that the traffic load increases.

Specifically, each sub-beacon is associated with a sending probability computed with an independent LCG with initial seed equal to the current value of the random generator of the inter-beacon times. Additionally, a sub-beacon transmission threshold $t h_{\mathrm{b}}$ is computed so that only sub-beacons whose associated sending probability, as per the LCG, is greater that th $h_{\mathrm{b}}$ are actually transmitted. This threshold is calculated so that, on average, $f-1$ sub-beacons are actually transmitted, so $t h_{\mathrm{b}}=1-(f-1) / n_{\mathrm{b}}$, where $f$ stands the speeding factor and $n_{\mathrm{b}}$ is the average number of sub-beacons between two regular beacons. The actual transmission time $b_{i}^{j}$ of the $j$-th subbeacon in the $i$-th interval, for $j=1, \ldots, k_{i}$, is $B_{i}+j \cdot \Delta_{B} / n_{\mathrm{b}}$ where $B_{i}$ denotes the transmission time of the previous regular beacon, $\Delta_{B}=\mathrm{E}\left[B_{i+1}-B_{i}\right]$ is the average cycle length, and $k_{i}$ is such that $B_{i}+k_{i} \cdot \Delta_{B} / n_{\mathrm{b}}<B_{i+1} \leq B_{i}+\left(k_{i}+1\right) \Delta_{B} / n_{\mathrm{b}}$. The overall scheme is depicted in Fig. 1 that shows the variation of the transmission threshold, the fixed length inter subbeacons length, and how only sub-beacons with an associated probability greater than the threshold are actually transmitted, represented with bold lines.

\section{B. Choosing the Beacon Rate}

Receivers can use the generation of sub-beacons to add more transmission opportunities for the senders. There remains, however, the problem of determining how many sub-beacons should be added between each pair of regular beacons.

We propose to maintain an estimation of the incoming rate $\lambda$ at each receiver in order to choose the optimum number of extra beacons. Such estimate could be calculated, for instance, with an exponential moving average of the number of successful receptions in each regular cycle, but other methods are possible. Assuming that the number of packets per cycle is known, we can proceed to calculate the beacon multiplication factor $f$. Note that $f=\lambda$ is generally not the best value, as there is an asymmetry between the cost of a failed transmission (collision) and that of an unanswered beacon.

1) Deriving the Optimum Beacon Rate: For obtaining the optimum multiplication factor with respect to energy consumption, we will first calculate the average amount of energy wasted by a given receiver and all its neighbor nodes with backlogged traffic to the receiver in a cycle, where a cycle is the time between two consecutive primary beacons. There are two sources for such energy expenditure: transmission collisions and unused beacons. Let us consider collisions first.

When there occur two or more simultaneous transmissions to a single receiver a collision happens and the energy $E_{\mathrm{b}}$ employed to transmit the beacon is wasted. ${ }^{1}$ Furthermore, the energy used by each sender listening while waiting for the beacon $E_{\mathrm{w}}$ and to actually transmit the data frame $E_{\mathrm{tx}}$ is also squandered. So, the amount of energy wasted by collisions in a cycle is

$$
E_{\mathrm{col}}^{1}(\lambda)=E_{\mathrm{b}}+\left(E_{\mathrm{w}}+E_{\mathrm{tx}}\right) \sum_{i=2}^{\infty} F(\lambda, i),
$$

where $\lambda$ is the average incoming rate at the receiver, measured in packets per cycle, $F(\lambda, i)$ is the probability mass function of the arrival distribution for the receiving node, and $i$ is the number of simultaneous transmissions in the cycle.

When the sub-beacons are in use, the cycle is split in $f$ sub-cycles and the total lost energy in the whole cycle is

$$
E_{\mathrm{col}}^{f}(\lambda)=f E_{\mathrm{col}}^{1}(\lambda / f) .
$$

\footnotetext{
${ }^{1}$ For the analysis, we assume a simplified worst case model where receivers cannot recover any frame whenever a collision happens.
} 


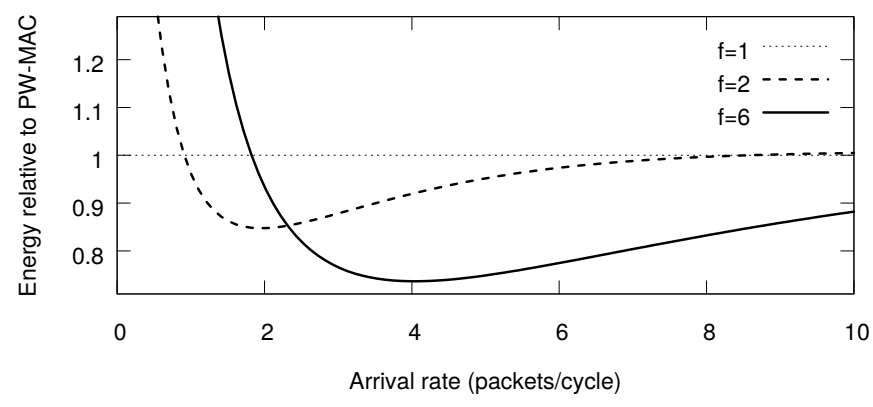

Figure 2. Energy waste, $E_{B}^{f}(\lambda)$, for different acceleration factors $f$ and varying arrival rate $\lambda$ compared to $\mathrm{PW}-\mathrm{MAC}(f=1)$.

However, as we reduce the cycle length, we are also increasing the beacon rate, and some energy is consumed sending unnecessary beacons. The amount of wasted energy due to that reason is given by

$$
E_{\emptyset}^{f}(\lambda)=f E_{\mathrm{b}} F(\lambda / f, 0) .
$$

So, the optimum multiplication factor $f$ is obtained when $E_{B}^{f}(\lambda)=E_{\text {col }}^{f}(\lambda)+E_{\emptyset}^{f}(\lambda)$ is minimum.

2) Optimum Beacon Rate for Poissonian Traffic: We can particularize the previous result for the special case of a Poisson distribution. Although in the edges of the network the distribution of time between frames in a given node is arbitrary, as we move towards the network core and traffic is aggregated the frame arrivals converge to a Poisson process, by virtue of the Palm-Khintchine theorem. Moreover, as we are mostly interested in calculating the number of extra beacons needed to adapt to this aggregated traffic, we can reasonably assume that nodes with extra beacons will receive, in fact, Poissonian traffic. ${ }^{2}$ So, substituting $F(\lambda, k)=\frac{\lambda^{k}}{k !} \mathrm{e}^{-\lambda}$ in (2) and (3) we get $E_{B}^{f}(\lambda)=f E_{\mathrm{b}}+\lambda\left(E_{\mathrm{w}}+E_{\mathrm{tx}}\right)+\mathrm{e}^{-\lambda / f}\left(f E_{\mathrm{b}}-\lambda\left(E_{\mathrm{w}}+E_{\mathrm{tx}}\right)\right)$. Now, finding the optimum $f$ is just a matter of deriving $E_{B}(\lambda, f)$ with respect to $f$ and solving

$$
\frac{\partial}{\partial f} E_{B}^{f}(\lambda)=E_{\mathrm{b}}\left(f+\mathrm{e}^{\lambda / f} f+\lambda\right) f-\lambda^{2}\left(E_{\mathrm{w}}+E_{\mathrm{tx}}\right)=0 .
$$

Unfortunately, there is no closed form for $f$ and resorting to numerical methods is out of the question for resourceconstrained sensors. For solving (4), we can take advantage of the fact that the minimum energy is consumed for values of $f$ close to $\lambda$, measured in packets per cycle. This is illustrated in Fig. 2, which shows the total energy waste $E_{B}^{f}(\lambda)$ versus $f$ for a range of traffic loads. The plotted values are normalized against PW-MAC, that corresponds with $f=1$, that is, without using sub-beacons. Therefore, we approximate $E_{B}^{f}(\lambda)$ by its second order Taylor series expansion around $\lambda$, assuming that the optimum $f$ value will be near the incoming rate

$$
\begin{aligned}
E_{B}^{f}(\lambda) & \approx \lambda \frac{(1+\mathrm{e}) E_{\mathrm{b}}-(1-\mathrm{e})\left(E_{\mathrm{w}}+E_{\mathrm{tx}}\right)}{\mathrm{e}} \\
& +\frac{\left(E_{\mathrm{w}}+E_{\mathrm{tx}}+E_{\mathrm{b}}\right)(f-\lambda)^{2}}{2 \mathrm{e} \lambda}
\end{aligned}
$$

\footnotetext{
${ }^{2}$ Albeit this assumption is invalid for high loads as the re-transmitted traffic is correlated, our method is able to accommodate higher traffic rates before collisions happen and thus the assumption remains valid for usual workloads.
}

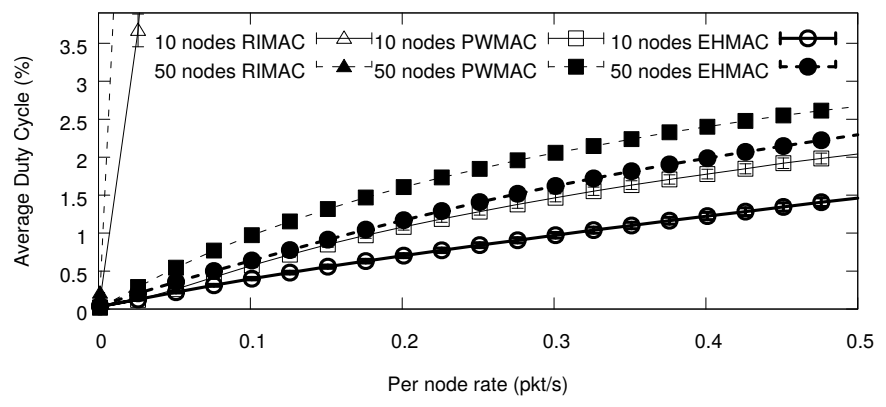

Figure 3. Duty cycle as a function of load.

$$
-\frac{\left(\frac{E_{\mathrm{w}}+E_{\mathrm{tx}}-2 E_{\mathrm{b}}}{\mathrm{e}}+E_{\mathrm{b}}\right)(f-\lambda)}{2 \mathrm{e} \lambda},
$$

derive (5) with respect to $f$ and solve. The final result is

$$
f^{*}=\lambda \frac{2\left(E_{\mathrm{w}}+E_{\mathrm{tx}}\right)-(1+\mathrm{e}) E_{\mathrm{b}}}{E_{\mathrm{b}}+E_{\mathrm{w}}+E_{\mathrm{tx}}}
$$

where all the terms are known in advance except $\lambda$, which is directly measured by the nodes.

\section{RESULTS}

We have experimentally verified that EH-MAC improves the original PW-MAC in the key performance metrics: delivery delay, sensor duty cycle, delivery reliability and collision probability. To this end, we have simulated a representative set of deployment scenarios. ${ }^{3}$ To serve as a reference point, we also tested the performance of the original RI-MAC [2] algorithm.

The parameters used in the numerical experiments were as follows. The main beacon interval at each node independently follows an uniform distribution between $500 \mathrm{~ms}$ and $1500 \mathrm{~ms}$ as in the original PW-MAC. The maximum sub-beacon rate has been capped to one sub-beacon every $100 \mathrm{~ms}$, so the maximum long-term sustainable rate at the receiver is 10 packets/s. Each EH-MAC receiver selects the beacon rate according to (6) and estimates $\lambda$ with a moving window of the last 15 inter-arrival times. Senders have been set to wake up $10 \mathrm{~ms}$ in advance to the predicted beacon transmission to account for the clock drift. Finally, the packets are 128 bytes long and the transmission rate is $250 \mathrm{~kb} / \mathrm{s}$, whereas beacons are 60 bits long. All the simulations were carried out on a $100 \mathrm{~m} \times 100 \mathrm{~m}$ field where nodes were randomly placed according to a spatial Poisson distribution of a given homogeneous density. To account for the fact that most traffic in the network goes towards a single sink node, a random node was chosen as the delivery destination. Traffic was then transmitted to it via multiple-hops using a greedy routing algorithm. The transmission distance was set to $35 \mathrm{~m}$. Every node generates traffic as a Poisson process with the same fixed rate. Every experiment was run for 1000 seconds and repeated 100 times varying the seed of the random generator of the simulator and the chosen sink. $95 \%$ confidence intervals of every metric were also calculated.

\footnotetext{
${ }^{3}$ The code for the simulations is available for download at https://migrax github.io/EH-MAC.
} 


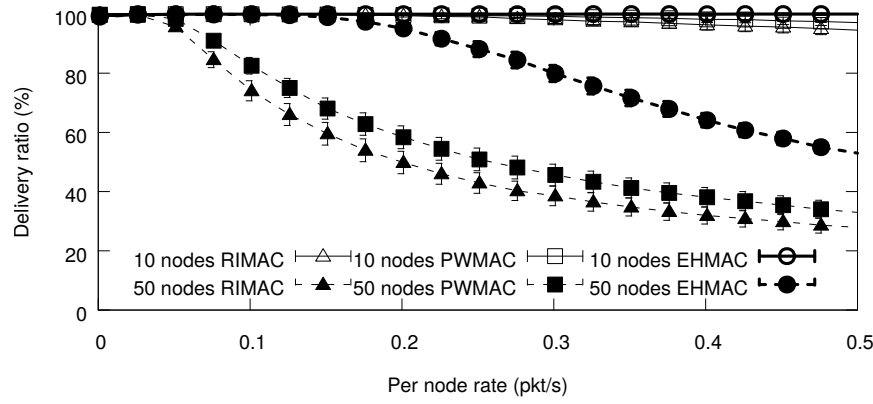

Figure 4. Delivery reliability as a function of load.

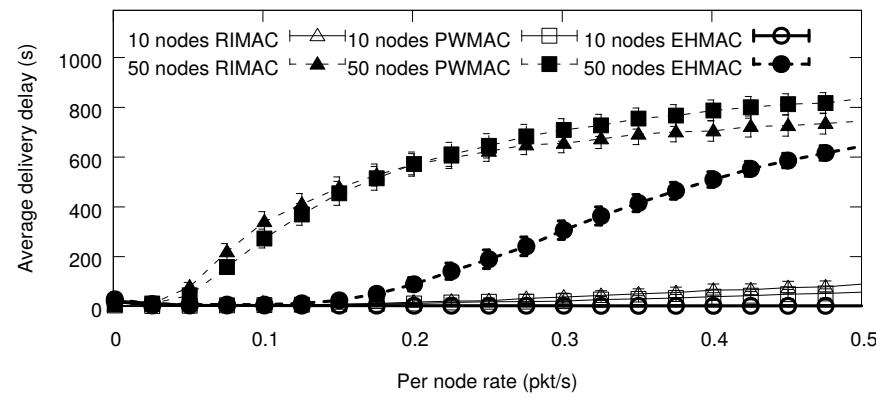

Figure 5. Average packet delivery delay as a function of load.

Figure 3 shows average duty cycle of the network nodes for the three MAC algorithms compared and two representative node densities. The average duty cycle, being the percentage of time that sensors have to keep their radios on, is ultimately responsible for the MAC protocol power consumption. The traffic rate was varied between 0 and 0.5 packets $/ \mathrm{s}$. Please note that this corresponds with a 5 packets/s rate at the receiver for the 10 nodes scenario and 25 packets/s for that with 50 nodes, so collisions are likely to occur near the sink. We clearly see that both PW-MAC and EH-MAC significantly outperform the original RI-MAC, while, at the same time, EH-MAC has an slightly better performance than PW-MAC.

The successful delivery ratio is plotted in Fig. 4. In the ten nodes setting, we see that all the protocols achieve an almost perfect reliability, although both RI-MAC and PW-MAC loose some packets at the highest loads. EH-MAC, on the contrary, is able to increase the beacon rate to accommodate the needs of the senders, achieving the perfect reliability even at the highest loads. For the 50 nodes case, the results get worse. When the rate reaches 0.02 packets/s, the effective rate at the receiver reaches 1 packet/s, the maximum both RI-MAC and PW-MAC were configured to support, and packets start to be discarded. In contrast, EH-MAC is stable until the generation rate reaches 0.2 packets $/ \mathrm{s}$, one order of magnitude greater. At that point, its performance also starts to diminish but, in any case, it stays considerably better than that of PW-MAC.

The average packet delivery delay, represented in Fig. 5, shows a similar trend to the delivery ratio. For the 10 nodes case the three algorithms show a good behavior, with slightly better results for our proposal. The improvement is much greater in the 50 nodes scenario, where it is clearly shown that EH-MAC copes with higher incoming rates until the maximum

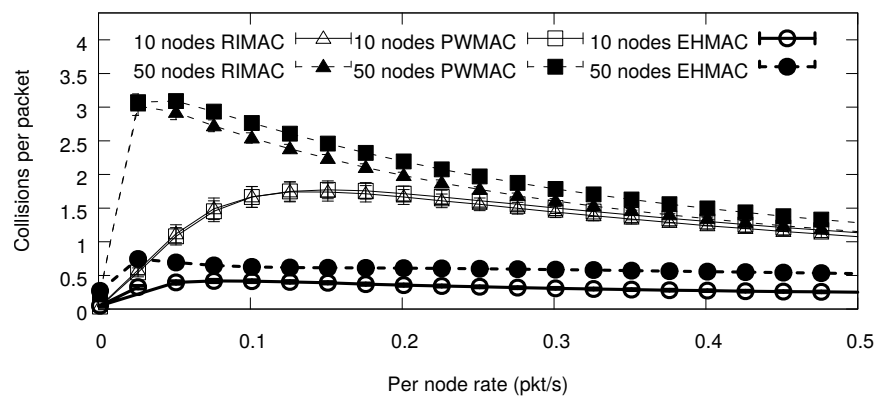

Figure 6. Number of collisions as a function of load.

is reached and the system cannot admit all the offered load. Note that the maximum delay is about 900 seconds because the simulations were limited to 1000 seconds, so no delivered packet can show higher delays. Unfortunately, this also gives the false impression that the delay asymptotically converges to a maximum value, whereas in reality either the delay would keep increasing unboundedly, or more packets would be lost.

The last figure shows the total number of collisions. Both RI-MAC and PW-MAC behave similarly, with more collisions than our enhanced EH-MAC in both settings, as shown in Fig. 6. But, as the load grows, the number of collisions decreases. This is because for high load many nodes are retransmitting collided packets, ultimately enlarging the time receivers wait for packets. For the enhanced algorithm, the effect is far less noticeable, because the algorithm adjusts the beacon rate so as to fit the demand of the senders. Otherwise, for the highest loads, collision retransmission comes up like in RI-MAC and PW-MAC.

\section{CONCLUSIONS}

We have proposed EH-MAC, a rate-adaptive mechanism for beacon generation in received initiated MAC protocols. EH-MAC produces an optimum number of new predictable transmission beacons while maximizing energy savings. The generation of new beacons does not have a performance impact on those senders unaware of them. We have compared via simulation the performance of EH-MAC against PW-MAC, obtaining better results in all important metrics.

\section{ACKNOWLEDGMENTS}

Work supported by the European Regional Development Fund (ERDF) and the Galician Regional Government under agreement for funding the Atlantic Research Center for Information and Communication Technologies (AtlantTIC).

\section{REFERENCES}

[1] E.-Y. A. Lin, J. M. Rabaey, and A. Wolisz, "Power-efficient rendez-vous schemes for dense wireless sensor networks," in IEEE ICC, vol. 7, Jun. 2004, pp. 3769-3776.

[2] Y. Sun, O. Gurewitz, and D. B. Johnson, "RI-MAC: a receiver-initiated asynchronous duty cycle MAC protocol for dynamic traffic loads in wireless sensor networks," in SenSys'08, Raleigh, Nov. 2008, pp. 1-14.

[3] X. Fafoutis, A. Di Mauro, M. D. Vithanage, and N. Dragoni1, "Receiverinitiated medium access control protocols for wireless sensor networks," Computer Networks, vol. 76, pp. 55 - 74, Jan. 2015.

[4] L. Tang, Y. Sun, O. Gurewitz, and D. Johnson, "PW-MAC: An energyefficient predictive-wakeup MAC protocol for wireless sensor networks," in Proceedings - IEEE INFOCOM, Shanghai, Apr. 2011, pp. 1305-1313. 